\title{
A Fuzzy Expert System Architecture for Intelligent Tutoring Systems: A Cognitive Mapping Approach
}

\author{
Mohammad Hossein Fazel Zarandi ${ }^{1}$, Mahdi Khademian ${ }^{2}$, Behrouz Minaei-Bidgoli ${ }^{3}$, \\ Ismail Burhan Türkșen ${ }^{4}$
}

${ }^{1}$ Department of Industrial Engineering, Amirkabir University of Technology, Tehran, Iran; ${ }^{2}$ Department of Computer Engineering \& IT, Amirkabir University of Technology, Tehran, Iran; ${ }^{3}$ Department of Computer Engineering, Iran University of Science and Technology, Tehran, Iran; ${ }^{4}$ Department of Industrial Engineering, TOBB Economy and Technology University, Ankara, Turkey.

Email: \{zarandi, khademian\}@aut.ac.ir,b_minaei@iust.ac.ir, turksen@mie.utoronto.ca

Received January $26^{\text {th }}, 2011$; revised September $25^{\text {th }}, 2011$; accepted October $9^{\text {th }}, 2011$

\begin{abstract}
An Intelligent Tutoring System (ITS) is a computer based instruction tool that attempts to provide individualized instructions based on learner's educational status. Advances in development of these systems have rose and fell since their emergence. Perhaps the main reason for this is the absence of appropriate framework for ITS development. This paper proposes a framework for designing two main parts of ITSs. Besides development framework, the second main reason for lack of significant advances in ITS development is its development cost. In general, this cost for instructional material is quite high and it becomes more in ITS development. The proposed method can significantly reduce the development cost. The cost reduction mainly is because of characteristics of applied mapping techniques. These maps are human readable and easily understandable by people who are not aware of knowledge representation techniques. The proposed framework is implemented for a graduate course at a technical university in Asia. This experiment provides an individualized instruction which is the main designing purpose of the ITSs.
\end{abstract}

Keywords: Concept Mapping; Expert and Student Models; Fuzzy Cognitive Maps; Intelligent Tutoring Systems; Expert Systems

\section{Introduction}

The idea of using computer for education goes back to 40 years ago with the establishment of Advanced Research and Projects Agency Net (Arpanet). Contrary to the purpose of the Arpanet, this network is also used for academic purposes. The reason for this is that the building blocks of the network were universities and academic centers in the United States. Current electronic learning systems have been utilized since 20 years ago with the development of internet protocols. An individual learner on the World Wide Web needs the following instructional helps and supports [1]:

- Access to learning materials;

- Strategies for learning;

- Time to learn;

- Advices on what to learn;

- Feedback on progress;

- Involvement and interactivity.

First generation electronic learning systems can only satisfy the primary needs of the individual learner, "Access to learning materials," while the others require more advanced learning systems (good to be named teaching systems).

By definition, intelligent tutoring systems (ITSs) are computer based instructional systems that attempt to gather information about a learner's learning status and having this information try to adapt the instruction to fit the learner's needs. Based on the definition, ITSs try to satisfy all needs of an individual learner, especially with personalization and individualized instruction. However, cost of ITS development is relatively high [2]. According to [3], development of one hour of instruction in KAFITS (an ITS) requires 100 hours of human work and as estimated by Woolf and Cunningham [4] this becomes to the ratio of 200:1 in average in ITS development, thus methods and framework for rapid development of ITSs are significantly helpful to increase utilizations of these systems in the world. For example, recent effort tries to simplify expert modeling by use of natural language [5]. Or create Example-Tracing Tutors without programming and just by drag and drop techniques which reduces development cost by a factor of 4 to 8 [6]. There are other efforts for creating ITSs efficiently [7-10] in the recent years. 
This paper introduces a new method for rapid development of ITSs using two well-known cognitive mapping techniques, fuzzy cognitive maps and concept mapping, and then presents the results of the proposed method which was experienced in topics relates to two sessions of a fuzzy course at a technical university in Asia. The rest of paper is organized as follows: Section 2 presents the backgrounds of the research. The proposed framework is presented in Section 3. The implemented system based on the proposed framework with its results is presented in Section 4. Finally, the conclusions and future works are presented in Section 5.

\section{Background}

\subsection{Architecture of an ITS}

There is no standard architecture for Intelligent Tutoring Systems (ITSs). However, past experiences suggest four emerging subsystem for an ITS [11]: Expert Model, Student Model, Pedagogical Module, and Communication Module which are depicted in Figure 1.

Student model stores information about user experiences during working with the system, such as the status of viewing contents, perceptions about degree of understanding of the user, and his/her last activities.

Expert model represents knowledge about the domain which intends to be presented for the learner. By using expert model, system makes judgment about learner's misconceptions and reasons for these mistakes.

Pedagogical module plays a significant role in implementing instruction method. By changing pedagogical module, the way of teaching can be completely changed. For example, teaching for K12 students (kindergarten through twelfth grade) or teaching in higher education is different from adult learners in many aspects of instruction (This results in a transition from pedagogical to andragogical approach). Pedagogical module primarily uses the knowledge of expert model and student model for its teaching purposes and decisions and actions of the pedagogical module relies on this provided knowledge. Thus, the more accurate and complete expert and student models results in more precise and effective actions of pedagogical module.

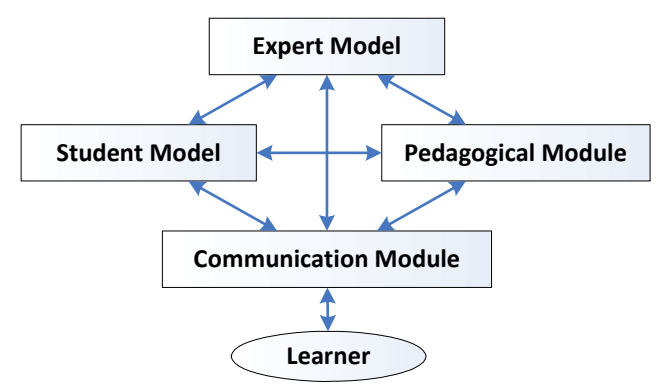

Figure 1. Four components of an ITS.
Finally, communication module deals with content presentation and capturing students' information during the learning sessions.

\subsection{Concept Maps}

Concept map is a graphical tool for visual representation of knowledge in an organized structure. Figure 2 shows the key features of a concept maps. The nodes of the map contain the concepts and the links between the nodes represent the relationship among these concepts.

Concept maps are widely used in various domains, especially in education; applications such as collaborative learning, learner evaluation and curriculum planning $[12,13]$. This is because of theory underlying concept maps and models about how humans learn new things based on the old structure of knowledge. Here, the key point is that the knowledge is stored in a networked structure and thus, the more organized the network results in more likelihood in recalling stored information in the learner's mind.

\subsection{Fuzzy Cognitive Maps}

Fuzzy cognitive maps (FCMs) are fuzzy graph structures for showing causalities between objects. While their fuzziness allows defining fuzzy degrees between fuzzy objects, their graph structure allows systematic causal propagation through the network and also knowledge base expansion by connecting multiple FCMs [14]. These graphs are developed based on prior work of political scientist, Robert Axelrod (1982), on cognitive

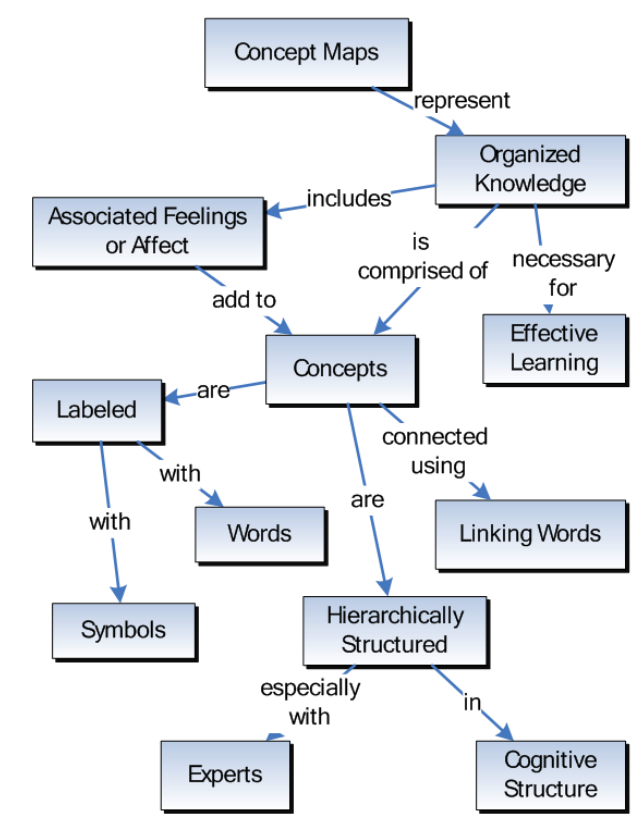

Figure 2. A concept map describing key features of concept maps. Concept maps are usually read from the top descending [12]. 
maps for representing social scientific knowledge [15]. The main difference between cognitive maps and FCMs is the use of linguistic variables to define causality between objects. A simple fuzzy cognitive map related to automobiles' accident is illustrated in Figure 3 (extracted from [16]). The Fuzzy Cognitive Map nodes are Auto Accidents, Patrol Frequency, Bad Weather, Own Risk Aversion and Freeway Congestion, and the system output is Own Driving Speed. Relations between the concepts or FCM's nodes are causal edges and also are fuzzy values. An example for causal relation is "Bad Weather" Usually increases "Automobile Accidents". Both edges and concept values could be expressed by fuzzy values, represented with membership functions. In this example the causality (the edge) is expressed in the forms of linguistic terms and measuring "Bad Weather" or other concepts (the concept values) could be done by fuzzy variables.

"The fuzzier the knowledge representation, the easier the knowledge acquisition and the greater the knowledge-source concurrence" [14]. This statement becomes more important in soft domains in which system concepts and relationships are basically fuzzy. Examples of soft domains are: political sciences, military, history and education. In addition, use of fuzzy knowledge representation helps us to modify or "tune" our system later just by modifying membership functions.

\section{The Proposed Model}

This section explains the proposed aggregated model of

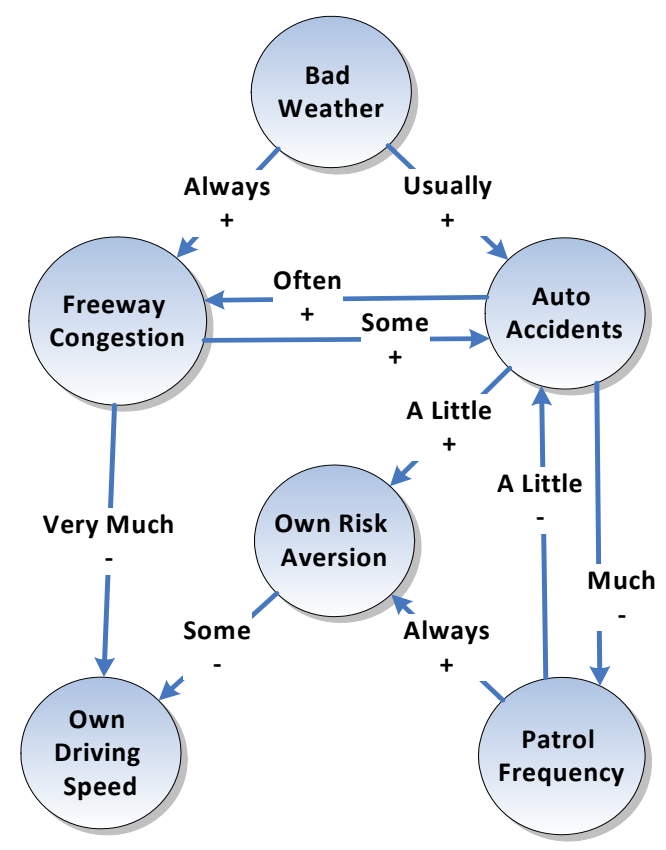

Figure 3. A fuzzy cognitive map which relates some driving concepts, such as speed, road congestion and accident [16].
ITS. Using the proposed model leads to a repaid development and consequently reducing production cost of ITSs. This rapid development is mainly because that using cognitive mapping techniques is so simple and easy for expert and then student modeling; these maps are expressive and human readable. They easily can be understood by the domain experts who are not familiar with more sophisticated knowledge representation techniques.

\subsection{Expert Model}

Concept maps have been used in various fields of education. This paper develops a different application for them. In the prior experiences, educational knowledge explicitly appears in these maps but here a concept map visualizes the structure of knowledge to be instructed later. In other words it works as a meta-knowledge container. This form of mapping sometimes is called knowledge structure map [1]. A simple concept map with prerequisite relation type is depicted in Figure 4. This map shows that playing chess requires that the player knows how chess pieces move. "How Chess Pieces Move" concept is related to "Play Chess" concept with "is prerequisite for" stereotype. We call this kind of concepts as instructional concept and for each domain of instruction, a map of instructional concepts should be defined to cover all required instructional materials for an individual learner; even elementary instructional concepts in contrast to the learner's advanced instructional objective.

Flexibility of the concept maps in defining concepts and ability to stereotyping them makes these maps as an ideal tool in expert modeling for ITSs. New concept types like questions and examples with their relationships with instructional concepts, "assessed by" and "exemplified by," may expand expert model to complete its design purposes which is to be explained in the student model part.

According to the general model of ITSs, expert model should assess learners' understandings and try to diagnose and reveals causes which might be the reason for his/her misconceptions. Assessment concept types such as placement assessments, diagnostic assessments and formative assessments perform these tasks ${ }^{1}$. One assessment concept in the concept map is capable of assessing multiple instructional concepts with different degrees of influence. When the learner fails to answer an assessment

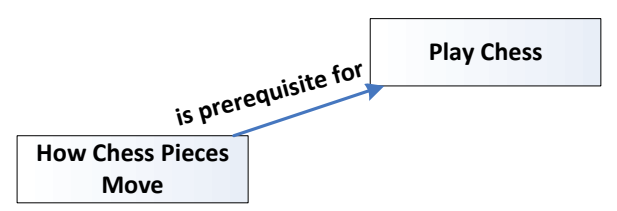

Figure 4. A simple concept map.

\footnotetext{
${ }^{1}$ Appendix 1 of this paper describes four types of assessments.
} 
concept, its negative effect propagates through the whole network by its defined relations and on the other hand his/her success, leads to increasing in system's belief of his/her degree of understanding.

Concepts in the concept map are systematically described with the use of static and dynamic properties. Hyperlink address of electronic contents associated with the instructional concepts is one example of the static properties. Dynamic properties hold dynamic information of the concepts and these properties are used to upgrade the expert model for modeling the student (student model). The value of these properties altered by defined causal relationships between the concepts. In the following subsection, this kind of properties and the alteration mechanism are explained.

\subsection{Student Model}

The general architecture of ITSs suggests the student model for holding information about user experiences during his/her interaction with the system. This model updates during learning session and dynamic properties are appropriate containers for this dynamic information. For example, properties such as "is viewed" and "is completed" are changed when the learner started to view the instructional concepts and has completed reading the concepts. But how and when these properties are changed in the system?

An effective tool for modeling updates of dynamic properties is fuzzy cognitive map. FCMs are also called Qualitative Systems Dynamics or Fuzzy Rule Based Systems [17]. With FCMs a cause and effect network is defined over the concept map. This network is responsible for altering the value of dynamic properties of the concepts; a causal relationship is defined between an event of a source concept and a dynamic property of a target concept(s). For example, as shown in Figure 5, correct answer to "question 2" causes the increase of system's perception of "degree of understanding" of a specific learner for "Introduction to Fuzzy Relations" instructional concept. In this example, the source and target concept(s) are "question 2" and "Introduction to Fuzzy Relations" respectively; a question concept type and an instructional concept type. Moreover, the event is correct answer to "question 2" and the dynamic property is system's perception of "degree of understanding" for "Introduction to Fuzzy Relations" concept.

Up to now some concepts have been described: expert model, student model, concept maps, fuzzy cognitive maps, static properties and dynamic properties. But what is the exact relationship between them? Despite static properties, dynamic properties of concepts are assigned per each learner and the student model acts as a container for them. In other words, the student model is a data

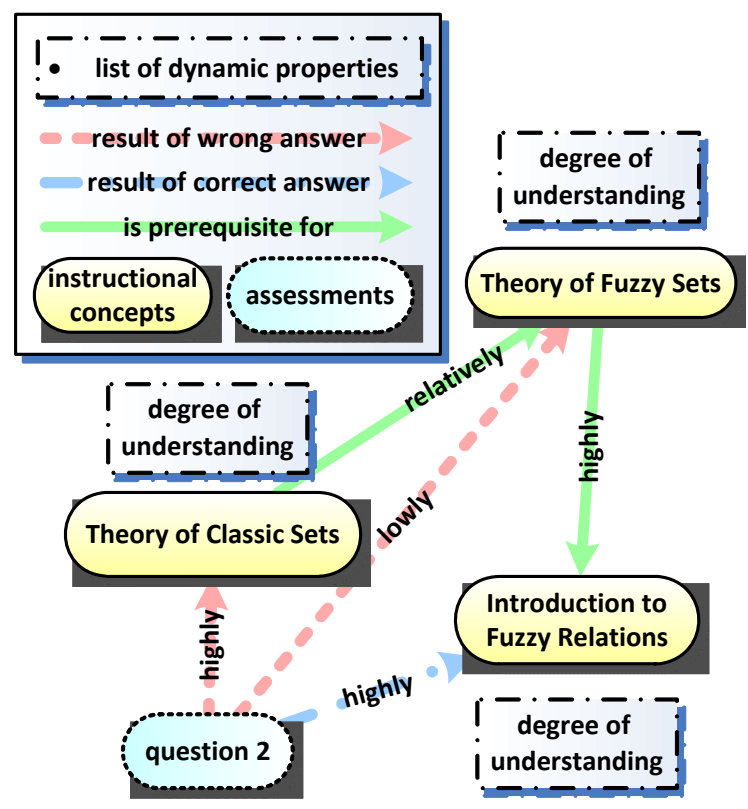

Figure 5. A simple exemplary expert model (and also student model-because of dynamic properties) for demonstrating causal relationship with two types of concepts: 1 ) Instructional concepts and 2) Assessments and three types of relationships: 1) Result of wrong answer, 2) Result of correct answer and 3 ) is prerequisite for.

structure for what is happened during the learning session and a fuzzy cognitive map is responsible for modeling and handling these changes. More precisely, in the proposed model, the student model is an instance of expert model which itself is built on a concept map and changing of dynamic properties of the concept map is happened by triggered events in the map and the amount of these changes is defined by causal relationships of fuzzy cognitive maps.

For each new student, expert model is instantiated with this new learner's ID and stored in the system's knowledge-base. In extensive expert models, the system can extract concepts which are related to learner's educational objective and only make use of these concepts for expert model instantiation. This extraction eliminates many irrelevant concepts with their relations and leads to a significant reduction in size of the expert model and consequently the student model. As a result, this reduction in size enhances the performance of system due to illumination of redundant processing.

Now the mechanism for assessing learners' understanding and diagnostic and remedial actions for solving his/her educational problems is described through the following example. In Figure 5, three instructional concepts are shown: "Theory of Classic Sets", "Theory of Fuzzy Sets" and "Introduction to Fuzzy Relations". The first concept is prerequisite for the second one and the third concept highly depends on the second one. 
Each instructional concept in this figure has just one dynamic property "degree of understanding” (DOU). DOU, for example can be measured and traced by a simple three staged variable or by a more sophisticated measurement such as Bloom's taxonomy of educational objective (More information about this taxonomy is provided in Appendix 2 of this paper). In addition to the concepts, Figure 5 has three rules and these are presented in the form of IF-THEN rules as:

- IF student's answer to "question 2" is wrong THEN DOU of him for "Theory of Classic Sets" will highly decrease;

- IF student's answer to "question 2" is wrong THEN DOU of him for "Theory of Fuzzy Sets" will slightly decrease;

- IF student's answer to "question 2" is correct THEN DOU of him for "Introduction to Fuzzy Relations" will highly increase.

When a learner faced with the "question 2" his/her response to this question triggers an event which leads to increase or decrease in system's perception of his/her DOU for the corresponding concept. After updating the value of the associated dynamic properties, the student model is updated and this leads to a new pedagogical decision which is the main responsibility of the pedagogical module which is discussed in the next part. The overall operation is shown in the Figure 7. For better understanding on how these alterations of DOU, as the most important dynamic property of educational concept, we should clarify two evidences during learning session: positive evidence and negative evidence.

Positive evidence. Positive evidence is defined in terms of any activity of learner which results in increasing the system's assumption of DOU of him/her. An example of this could be a correct answer of learner to a proposed question. Each positive evidence is characterized by the level of Bloom's taxonomy and a confidence level. Positive evidences in proposed system are:

- Correct response of learner in assessments: When learner answers the proposed questions correctly, the system's assumption of DOU of him should be increased to the specified level of Bloom's taxonomy with respect to relation's confidence level.

- Learner's study of electronic content of educational concepts: After reading electronic content by a learner, the system's assumption of DOU of him should be increased by the specified Bloom's taxonomy level which is defined inside of electronic content. Here, the level of confidence is calculated based on reading allocated time.

- Learner's study of examples of educational concepts: Same as learner's study of educational concept, learner's study of examples also results in increasing in his DOU in all educational concepts which relates to the example. Moreover, level of confidence also is calculated based on reading allocated time.

Negative evidence. Conversely, any activity of learner which results in decreasing the system's assumption of DOU of him called negative evidence. Wrong response of learner in assessments is the only defined negative evidence in the proposed system.

\subsection{Pedagogical Module}

Based on the gathered information by the student model, pedagogical module proposes comprehensive instruction in agreement with the learner's need. Most of the ITSs' pedagogical module operates in the form of procedural rules [11]. These rules might be fired on certain student actions or on recognized student model situations which are defined in the rules. Pedagogical module of the proposed system is designed based on subsumption architecture [18] in which some instructions' priority suppresses the others from operation. For instance, in a mobile robot in robot path planning, instructions for obstacle avoidance are much more important than instructions for following the target. For an example in the educational domain, instructions for satisfying prerequisite type relation are more important than instructions for offering new educational concepts to the learner. Pedagogical module of the proposed system consists of five layers of instructions in a subsumption architecture style. Before description of each layer, three kinds of educational concepts should be defined for clarification (according to Figure 6).

Satisfied educational concepts (group 1) are educational concepts which all of requirements are fully satisfied. In Figure 6, "addition" concept is one example for satisfied educational concept because it requires comprehension level of "understanding meaning of numbers" which is currently in application level and application level is one layer above comprehension level. In this figure, "signed numbers" is not a satisfied concept, since it requires higher level of understanding in "addition" and "subtraction".

- Educational concepts which have fully satisfied their subsequent concepts (group 2) are concepts that their subsequent concepts do not need higher level of understanding of them. In Figure 6, "understanding meaning of numbers" is an example of this group as a result of its current DOU of application level and subsequent concepts ("addition" and "subtraction") with the need of comprehension level.

- Educational concepts which are candidates for presentation (candidates): We define these concepts as concepts which are ready for presentation and extraction of them is done by the following method. Set of group 2 concepts subtracts from set of all concepts in group 1; result is candidates set. 


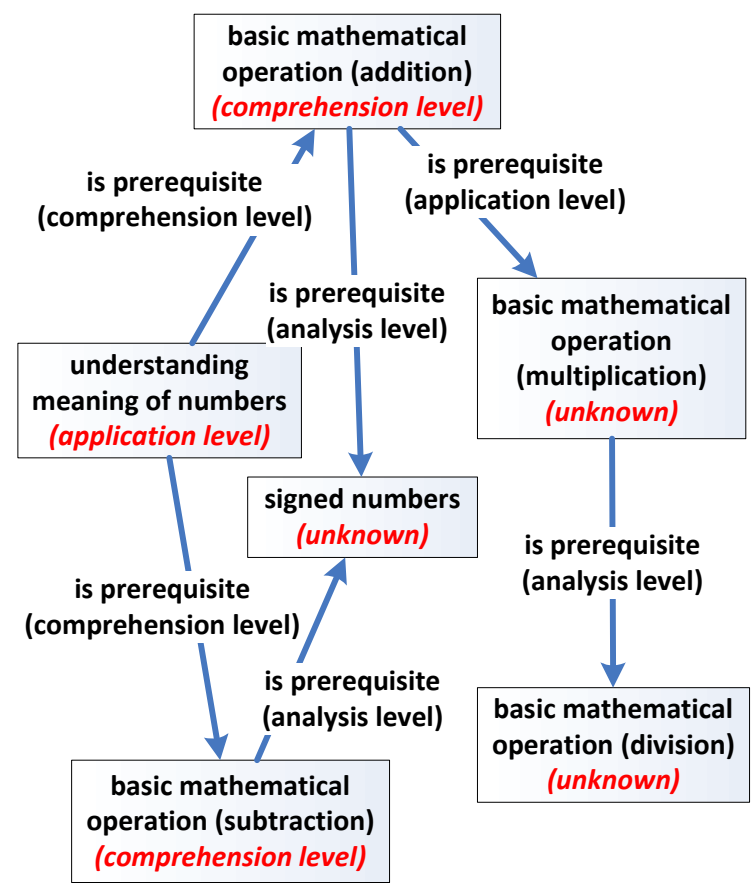

Figure 6. A simple student model (an instance of expert model).

In any cycle of learner interactions with the system, learner specifies an educational concept as learning objective. Then system extracts all required educational concepts and their related other concepts in the expert model. Based on this extraction, a new student model is instantiated and can be added to the old student model. Information in the old student model helps pedagogical module to prevent instructing previously learner known materials. Now the goal of pedagogical module is to take the learner to his chosen learning objective. For this purpose, pedagogical module uses the following layers of instruction (in a cycle of instruction, if student model meets the criteria of a specific layer then the instructions of that layer followed and pedagogical module stops at that layer):

1) If there is no candidate in the candidates set, then the learner is reached to his educational objective.

2) Select (and then inquire) the most preferred placement assessment from set of unanswered placement assessments which has relationship with not shown candidates (from the candidates set). The most preferred placement assessment is the one with maximum relationship level to all related educational concepts. The main goal of this step is to eliminate system from presenting already known materials to the learner.

3) Select (and then show) the most preferred educational concept from not shown candidates of the candidates set. Here, the most preferred concept has the maximum need of DOU to become as a group 2 concept. At this level, Primary learning is done because the new educational concepts are presented to the learner.

4) From examples related to previously displayed candidates, select (and then show) the most preferred one. Here, the most preferred example has the maximum positive evidence in related educational concepts. The aim of this level is to present additional illustration for educational concept to upgrade them into group 2 concepts.

5) From unanswered formative assessments which have relation with previously displayed candidates, select (and then ask) the most preferred one. The most preferred assessment has the maximum relationship with educational concepts. This level assesses the learner and adapts instruction to meet his needs. Adaptation in instruction is done by altering DOU in related educational concepts which itself is the result of positive and negative evidences of assessment. After changing in DOU, group 1, group 2, and as the result, candidates set are modified.

6) Selection of assessments, examples, or educational concepts offered to the learner.

According to the strategy of tutoring system, learner, at any time, could choose learning materials and assessments himself which leads to updating the student model. Updates in student model causes changing in elements of group 1, group 2, and then candidates set which means an adaptation according to learner's action.

Pedagogical module serves other actions during learning session. One important example of this is "termination suggestion". If a learner allots more time than specified required time for an educational concept, the system suggests termination. If the learner terminates reading the educational concept, "is viewed" status will be altered to true. In next cycle of operation of pedagogical module, another educational concept might be nominated for presentation (operation of level 2) or an example for previous educational concept, if it is available, will be presented (operation of level 3).

\subsection{Communication Module}

In the proposed system, communication module has two responsibilities. It visualizes student and expert model for learners and captures environment variables like learner's allotted time for studying a specific content. When a learner finishes his/her studying of a specific content this environment variable acts as an event and changes the dynamic property of related concept(s) which means update in student model. Pedagogical module by use of the updated student model presents new educational material for the learner, offers teaching suggestions or assesses the learner. After this, the student model updates again and this cycle continues. Figure 7 illustrates the overall operation of the system. 


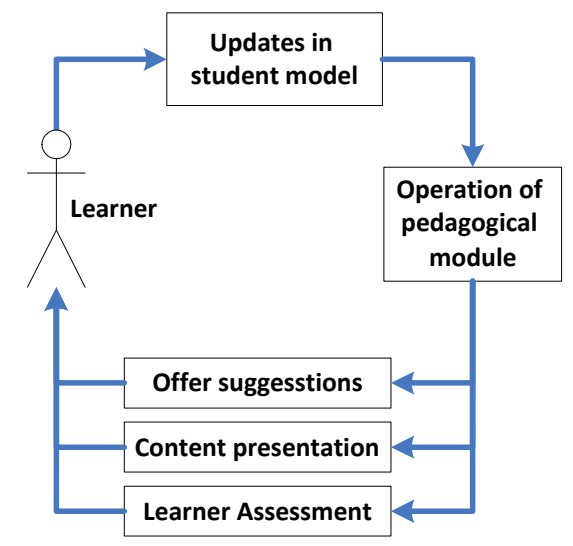

Figure 7. The overall operation of system.

\section{Implementation and Results}

Implementation of the system and its properties based on proposed model is described in this section. For expert and student models, the domain of instruction for ITSs impact on selecting types of concepts, properties and relations, however, this difference is not as much as one which is dominated in designing other advanced teaching/learning systems like cognitive tutors. This is the reason why other types of advanced teaching/learning systems like cognitive tutors are domain based and could not be made in a general domain. Therefore, the expert team should decide on types of concepts, properties and relations and grouping them into essential elements. In the proposed system, four types of concepts are defined: instructional concepts, placement assessments, formative assessments and examples. Two types of causal relationship and one simple stereotype relates the concepts to each other. All concepts have two common dynamic properties, "is viewed" and "is completed," and instructional concepts have another dynamic property by title of "degree of understanding." Degree of understanding is adjusted by causal relationships which exist between assessments, examples, and concepts. Concepts also contain five static properties: title, color, position, radius and hyperlink. First four static properties are used for visual representation purposes in the communication model and the last one, the hyperlink, is used for content presentation.

Pedagogical module is designed based on Brook's architecture [18] and consists of five suppressive layers. By applying the expert and student models, suggested materials and instructions are individualized for learners and each learner is able to experience a different navigation path regarding the others. Six layers of instruction method in the pedagogical module are described in section 3.3 of this paper (Pedagogical Module). In these six layers the most preferred concept selected by calculations on its relation degree with related concepts. Detailed calculations for this most preferred concept selection is not presented here because it is outside the scope of this paper and the implemented pedagogical module is just used as an example in our experienced system.

Based on the strategy of tutoring system (pedagogical module), the learner, at any time, is able to choose learning materials and assessments, himself which leads to update in the student model and consequently resuming systems work (Look at Figure 7).

Our system can facilitate communications by the use of Microsoft Silverlight and Microsoft Agent technologies. Using the Silverlight technology the student model is visually represented in a colored graph with fuzzy links and various shapes and the learner can submit his/ her request with this graph. Learner can also see his/her model anytime during his/her interactions with the system. Part of visual representation of the expert model for implemented system is demonstrated in Figure 8. In this figure three types of concepts are represented: instructional concepts, questions and examples and relations between them are demonstrated with fuzzy links. For example "butterfly example", a well-known example in the fuzzy clustering, is closely related to the "Fuzzy Clustering" and "Bezdek Algorithm" concepts. In this map, also, "question 12" is a question type concept which cannot significantly assess "Fuzzy Clustering" instructional concept but it can assess the "Crisp Clustering" concept. The whole experienced network consists of more than 40 concepts.

The pedagogical instructions and suggestions are demonstrated by an agent character with Microsoft Agent Technology and agent uses text to speech synthesizers

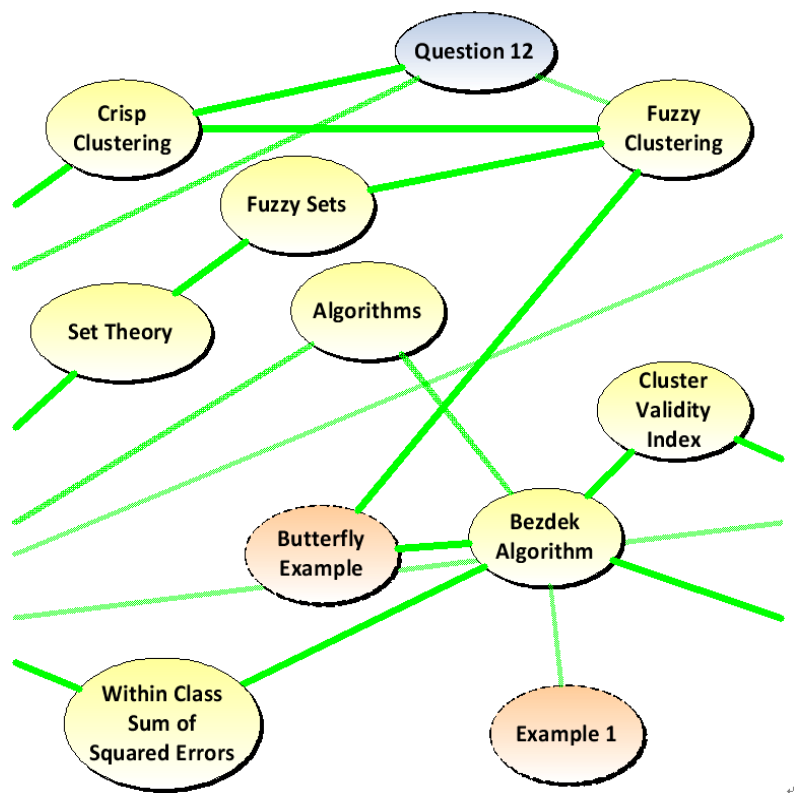

Figure 8. Extracted part of visual representation of expert model of the implemented system. 
(TTS Engine) for auditory communications. Agent also can hear learner's predefined requests and process learner's voice with its speech recognition Engine.

\subsection{First Prototype: Teaching Fuzzy Clustering}

The proposed ITS model in which expert and student models are created by cognitive mapping methods, is constructed for concepts are related to two sessions of graduate fuzzy course by the title of fuzzy clustering. The implemented system is an open web based ITS for learning fuzzy pattern recognition and fuzzy clustering. Elementary required instructional concepts such as the concepts with topic of mathematical sets, fuzzy sets and algorithms are embedded in the system which makes it an ideal tool for learning advanced graduate topics in fuzzy theory for undergraduate students.

Expert model's map of implemented system consists of 20 assessments, three examples and 20 instructional concepts. The whole network constructed with more than 200 well-defined relationships. Each assessment node has one related multiple choice question and can assess three concepts simultaneously in average. Examples and instructional concepts have their electronic resource of educational content in the form of web pages.

The pedagogical module has five layers of defined instruction method which is briefly defined in this paper and the communication module facilitates communication by the use of two technologies, Microsoft Agent and Silverlight technologies.

Construction of whole network and electronic concepts takes about 150 hours of human work and the system provides about 200 minutes of individualized instruction (with related prerequisites). This means that the ratio of 45:1 for system development in the selected graduate course is obtained. Even though this experience is just one instance, the course is an advanced graduate course and this is a significant time reduction in ITS development.

This time reduction mainly is due to the use of concept maps and especially fuzzy cognitive maps in dealing with this soft domain (education) for expert and student modeling. In this methodology, the experts of a specific domain are able to sketch the expert model and put this model into the implemented system, using the recommended pattern. Therefore, this approach also can reduce the development time of ITSs with no loss of productivity. Using this methodology, expert and student models are separated from the codes. Development of two other parts of the system and their expansion can be done with no hard-coding, but just by expanding these models themselves with use of a simple GUI application as an editor.

This methodology could also be applied for covering other fuzzy pattern recognition subjects, such as image processing and expert systems development with fuzzy clustering for future work. This expansion can be done just by defining and inserting the required concepts and the related electronic contents into the implemented system without any hard-coding.

\subsection{A Tutoring System for Teaching RLC Circuits}

After successful prototype of the proposed model in summer 2007, the authors decide to integrate the system with a CMS for establishing another real experiment of system in autumn 2008. This time a section of electric circuits' course by the title of "The Complete Response of Circuits with Two Energy Storage Elements" is selected for the expert model domain. The system attached to Masir CAMP, a content management system, which enables establishing a dynamic web-site with user registration, interaction with web-controls, establishing exams and surveys with advanced logging system. The expert model is designed with a graphical drag-drop tool and consists of about 45 nodes. In this experiment students of two groups of electric circuits' course are encouraged to attend and about 40 students were registered in the system and about 32 of them continuously and actively attended in the planned programs.

\section{Conclusions and Future Works}

In this paper, a methodology for designing expert and student models of an ITS with the use of cognitive mapping techniques has been presented. The aim of presenting this approach is to provide a systematic way of designing expert and student models of general domain ITSs.

The implemented system with its expert model was used for teaching two sessions of a graduate course and the course instructor never taught these materials again for the course students. Oral discussion with the students reveals that they are satisfied with this way of learning especially that they are able to navigate the contents and review them repeatedly while the system remembers each learner's status in their student models. The students also are able to read and practice the course problems and experience completely different sessions based on their needs. This experimental study shows that the proposed model is demonstrated to successfully improve efficiency of ITS construction that provides individualized instruction based on the learners' needs.

As it is mentioned in the result part of this paper, this method also was applied for a second experience related to electric circuits' course. The results of final survey of this experience shown that more than $95 \%$ of the students have benefited from the system and they believe that this experiment helps them in their final exam of their course. In addition $92 \%$ of them believe that the system works 
well on prerequisite consideration while about $80 \%$ of this group thinks that they experience different sequence of contents from their friends. The detailed questionnaire results are presented in the Appendix 3 of this paper.

As a final word, it is noticeable that the described methodology is in its childhood steps and it requires more and more investigation and experience. Although, the authors believe that this first step is able to speed up ITS development growth in the world. Moreover, the authors suggest the following developments as the future work and completion of this research:

- Expanding the expert model to support educational concepts in a hierarchical manner for including broader scientific domains. In other words, develop a model to define relationships between educational concepts in different levels in a hierarchy;

- Develop more advanced pedagogical module by applying advanced human behavior modelers such as SOAR, architecture for human cognition;

- Develop methods for extracting expert models in a collaborative manner in which experts of a specific domain work together to form an expert model collaboratively.

\section{Acknowledgements}

The authors would like to thank Masir Ltd. for its cooperation in integrating Masir CAMP and the implemented system in the second experience. In addition the authors gratefully acknowledge the support of electric circuits' expert S. J. Mousavi during the design of the expert model of the second experience and Mr. H. P. Sadjad for providing electronic contents. In this project some parts of electric circuits' and differential equation course materials of MIT open courseware were used which enriched the electronic contents. The authors thank the MIT open courseware for providing such great source of educational materials. Finally authors thank students who attended in our experience actively and energetically.

\section{REFERENCES}

[1] J. L. Gordon, "Creating Knowledge Maps by Exploiting Dependent Relationships," Knowledge Based Systems, Vol. 13, No. 2-3, 2000, pp. 71-79. doi:10.1016/S0950-7051(00)00048-4

[2] M. Moundridou and M. Virvou, "Analysis and Design of a Web-Based Authoring Tool Generating Intelligent Tutoring Systems," Computers \& Education, Vol. 40, No. 2, 2003, pp. 157-181. doi:10.1016/S0360-1315(02)00119-7

[3] T. Murray, "Authoring Intelligent Tutoring Systems: An Analysis of the State of the Art," International Journal of Artificial Intelligence in Education, Vol. 10, No. 1, 1999, pp. 98-129.

[4] B. P. Woolf and P. A. Cunningham, "Multiple Knowl- edge Sources in Intelligent Teaching Systems," IEEE EXpert, Vol. 2, No. 2, 1987, pp. 41-54.

doi:10.1109/MEX.1987.4307063

[5] S.-Y. Jung and K. VanLehn, "Developing an Intelligent Tutoring System Using Natural Language for Knowledge Representation," Intelligent Tutoring Systems, Vol. 6095, 2010, pp. 355-358. doi:10.1007/978-3-642-13437-1 69

[6] V. Aleven, B. M. McLaren, J. Sewall and K. R. Koedinger, "A New Paradigm for Intelligent Tutoring Systems: Example-Tracing Tutors," International Journal of Artificial Intelligence in Education, Vol. 19, No. 2, 2009, pp. 105-154.

[7] V. Aleven and J. Sewall, "Hands-On Introduction to Creating Intelligent Tutoring Systems without Programming Using the Cognitive Tutor Authoring Tools (CTAT)," Proceedings of the 9th International Conference of the Learning Sciences, Vol. 2, Chicago, 29 June-2 July 2010.

[8] S. Stankov, M. Rosić, B. Žitko, and A. Grubišić, "TExSys Model for Building Intelligent Tutoring Systems," Computers \& Education, Vol. 51, No. 3, 2008, pp. 10171217.

[9] W. R. Murray, "Statistical Relational Learning in Student Modeling for Intelligent Tutoring Systems," Artificial Intelligence in Education, Vol. 6738, 2011.

[10] P. Fournier-Viger, R. Nkambou and E. M. Nguifo, "A Knowledge Discovery Framework for Learning Task Models from User Interactions in Intelligent Tutoring Systems," Advances in Artificial Intelligence, Vol. 5317, 2008, pp. 765-778.

[11] B. P. Butz, M. Duarte and S. M. Miller, "An Intelligent Tutoring System for Circuit Analysis," IEEE Transactions on Education, Vol. 49, No. 2, 2006, pp. 216-223.

[12] J. D. Novak and A. J. Cañas, "The Theory Underlying Concept Maps and How to Construct Them," 2006. http://www.cmap.ihmc.us

[13] J. Novak and D. Musonda, "A Twelve-Year Longitudinal Study of Science Concept Learning," American Educational Research Journal, Vol. 28, No. 1, 1991, pp. 117153.

[14] B. Kosko, "Fuzzy Cognitive Maps," International Journal Man-Machine Studies, Vol. 24, No. 1, 1986, pp. 6575. doi:10.1016/S0020-7373(86)80040-2

[15] A. Konar, "Computational Intelligence. Principles Techniques and Application," Springer, Berlin, 2006.

[16] D. Brubaker. (1996, Apr.) EDN Access: For Design, By Design.

Uhttp://www.edn.com/archives/1996/042596/09column.h tmU

[17] J. P. Carvalho and J. A. B. Tomé, "Rule Based Fuzzy Cognitive Maps-Qualitative Systems Dynamics," 19th International Conference of the North American, Atlanta, 13-15 July 2000, pp. 407-411.

[18] R. A. Brooks, "A Robust Layered Control System for a Mobile Robot," IEEE Journal of Robotics and Automation, Vol. 2, No. 1, 1986, pp. 14-23. doi:10.1109/JRA.1986.1087032 


\section{Appendix 1. Types of Assessments}

In a process of teaching usually there are four types of assessments which are taking place; placement assessments, formative assessments, diagnostic assessments and summative assessments. New students are assessed by placement assessments in order to determine where they should start in the instructional sequence. Before starting a specific topic and especially during instruction, teachers use formative assessments to evaluate whether the topic is covered completely and accurately and if formative assessment does not reveals problems which may occur in certain instructional objectives, diagnostic assessments are used. This type of assessment involves diagnostic instruments as well as observation techniques and usually only one student is under investigation to determine his problems. Diagnostic assessment searches for root causes resulting in learning problems and offer remedial advices. Finally, summative assessments are taken at the end of an instructional unit to grade students and specify how well they have attained the instructional objectives. Educators believe that teachers commonly use this type of assessment while other tree types have significant effect for improving students' achievements in learning.

\section{Appendix 2. Bloom's Taxonomy of Educational Objectives}

In 1956, a group of educational psychologists and university examiners presented a classification system in learning. This classification is named Bloom's taxonomy of educational objectives. In this taxonomy, educational objectives are primarily categorized in three major classes: objectives in cognitive, affective, and psychomotor domains.

The goals in cognitive domain relates to intellectual skills involving acquisition, process, reasoning, and use of knowledge in application areas. Bloom's team identifies six classes in this domain which they are also divided into subclasses. Knowledge, comprehension, application, analysis, synthesis, and evaluation are six classes within the cognitive domain. Success in each class in this domain requires students' achievement in inferior classes. For example, successful utilization of specific knowledge never happens without its comprehension.

The affective domain mainly focuses on attitudes and feelings and is categorized into five classes: receiving, responding, valuing, organization, and characterization. For example, if a teacher is worried about learner's lack of interest in physics, his concern relates to affective domain.

The psychomotor domain deals with the physical activities of students. However, Blooms and his colleagues have not been made any taxonomy for this domain, but several taxonomies of this domain have been extracted over the years since the original books of Bloom.

Current intelligent tutoring systems mainly deal with the cognitive domain rather than the two others. This means that educational objectives of these systems might be designed with consideration of defined stages in the cognitive domain. A linear representation of stages in the cognitive domain is demonstrated in Figure 9. The expert and student models of the proposed system employ stages in the cognitive domain for measuring learners' understanding of the specific topic or educational material which will be explained in the next section.

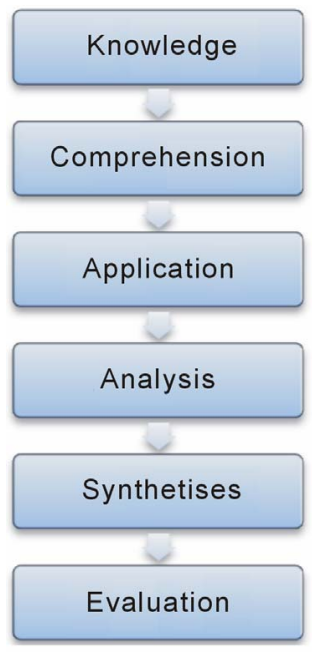

Figure 9. Linear representation of stages in cognitive domain. 


\section{Appendix 3. The Second Experience's Questionnaire and Its Results}

\begin{tabular}{|c|c|c|}
\hline Question title and choices & \# Total 26 & $\%$ \\
\hline $\begin{array}{l}\text { Your opinion about the exam's time and duration? } \\
\text { - Appropriate and adequate } \\
\text { - It needs more time }\end{array}$ & $\begin{array}{c}18 \\
7\end{array}$ & $\begin{array}{l}70 \% \\
27 \%\end{array}$ \\
\hline $\begin{array}{l}\text { Your opinion about the difficulty of the exam's questions? } \\
\text { - Hard } \\
\text { - Appropriate according to contents } \\
\text { - Easy }\end{array}$ & $\begin{array}{c}13 \\
13 \\
0\end{array}$ & $\begin{array}{c}50 \% \\
50 \% \\
0 \%\end{array}$ \\
\hline $\begin{array}{l}\text { Exam questions' relativity to system's content: } \\
\text { - They are quite relative. } \\
\text { - They are not designed uniformly according to content. }\end{array}$ & $\begin{array}{c}19 \\
7\end{array}$ & $\begin{array}{l}73 \% \\
27 \%\end{array}$ \\
\hline $\begin{array}{l}\text { Your opinion about the website accessibility and availability: } \\
\text { - It was available and accessible with suitable speed. } \\
\text { - It was unavailable in many times. } \\
\text { - The web-site was too slow. }\end{array}$ & $\begin{array}{c}18 \\
6 \\
2\end{array}$ & $\begin{array}{c}70 \% \\
23 \% \\
7 \%\end{array}$ \\
\hline $\begin{array}{l}\text { Your opinion about the user-interfaces: } \\
\text { - I feel well while working with the system. } \\
\text { - It has appropriate design by considering it as an academic project. } \\
\text { - It has a poor design. } \\
\text { - It has problem in my web-browser/operating system. }\end{array}$ & $\begin{array}{c}5 \\
7 \\
12 \\
2\end{array}$ & $\begin{array}{l}19 \% \\
27 \% \\
46 \% \\
8 \%\end{array}$ \\
\hline $\begin{array}{l}\text { Bandwidth requirement for multimedia contents: } \\
\text { - I didn't see multimedia contents. } \\
\text { - My experience was great and I have high speed internet connection. } \\
\text { - My experience was not bad and I have dial-up connection. } \\
\text { - My experience was bad and I have dial-up connection. }\end{array}$ & $\begin{array}{l}15 \\
8 \\
3 \\
0\end{array}$ & $\begin{array}{l}58 \% \\
31 \% \\
11 \% \\
0 \%\end{array}$ \\
\hline $\begin{array}{l}\text { Your overall opinion about the website: } \\
\text { - } \quad \text { Very good } \\
\text { - } \quad \text { Good } \\
\text { - } \quad \text { Not bad } \\
\text { - } \quad \text { Bad }\end{array}$ & $\begin{array}{c}2 \\
14 \\
10 \\
0\end{array}$ & $\begin{array}{c}8 \% \\
54 \% \\
38 \% \\
0 \%\end{array}$ \\
\hline $\begin{array}{l}\text { You opinions about the website's help and support: } \\
\text { - It was good. } \\
\text { - I have asked questions but I didn't get any answer from the support team. } \\
\text { - The support was good but there was latency in answers. }\end{array}$ & $\begin{array}{c}15 \\
1 \\
2\end{array}$ & $\begin{array}{c}58 \% \\
4 \% \\
8 \%\end{array}$ \\
\hline $\begin{array}{l}\text { Do you think you have benefited from the course? } \\
\text { - Yes. I think. } \\
\text { - I have benefited from some part of it. } \\
\text { - No, I haven't. }\end{array}$ & $\begin{array}{c}7 \\
17 \\
1\end{array}$ & $\begin{array}{c}27 \% \\
65 \% \\
4 \%\end{array}$ \\
\hline $\begin{array}{l}\text { Do you suggest other students to take the course? } \\
\text { - Yes } \\
\text { - Yes, definitely. But after the system improvement. } \\
\text { - No }\end{array}$ & $\begin{array}{c}9 \\
16 \\
1\end{array}$ & $\begin{array}{c}35 \% \\
61 \% \\
4 \%\end{array}$ \\
\hline $\begin{array}{l}\text { Do you think that taking this course may help you in final exam of your electri } \\
\text { - Yes } \\
\text { - } \quad \text { No }\end{array}$ & $\begin{array}{c}19 \\
7\end{array}$ & $\begin{array}{l}73 \% \\
27 \%\end{array}$ \\
\hline $\begin{array}{l}\text { Your opinion about the contents: } \\
\text { - Adequate and appropriate } \\
\text { - The contents of this course are too much. } \\
\text { - It needs more multimedia contents }\end{array}$ & $\begin{array}{c}10 \\
3 \\
10\end{array}$ & $\begin{array}{l}39 \% \\
12 \% \\
39 \%\end{array}$ \\
\hline $\begin{array}{l}\text { Do you think that the project would be succeeded in the future versions acc } \\
\text { objective (to be a real ITS)? } \\
\text { - Yes } \\
\text { - No }\end{array}$ & $\begin{array}{c}25 \\
1\end{array}$ & $\begin{array}{c}96 \% \\
4 \%\end{array}$ \\
\hline
\end{tabular}




\section{Continued}

Do you think that step-by-step learning helps students in learning educational subjects?

- Yes

- It depends on learning materials.

- No

$\begin{array}{cc}13 & 50 \% \\ 13 & 50 \% \\ 0 & 0 \%\end{array}$

Overall, how much interested have you been about the project?

- I am interested in the project.

- I take part in this activity because of its extra mark.

- It was a waste of time.

$\begin{array}{cc}16 & 62 \% \\ 10 & 38 \% \\ 0 & 0 \%\end{array}$

The early day's feedbacks show that the system works-well on prerequisite consideration for content viewing. Do you agree with this?

- Yes

- No. I saw materials which I didn’t pass their prerequisite contents.

Do you think that other students might saw contents in different sequence according to their answers to the placement assessments?

- Definitely no.

What is your overall opinion about the functionality of the system (as an ITS)?

- I think the system was good.

- I think it needs more improvements but it would be a great educational tool later.

- I think it was a failed project.

The last question, your opinion about this questionnaire?

- I think that questions are phrased wisely and precisely.

- It has too many questions.

- It doesn't have appropriate choices. 